\title{
ASSESSMENT OF FOUR TYPICAL TOPOGRAPHIC CORRECTIONS IN LANDSAT TM DATA FOR SNOW COVER AREAS
}

\author{
Yan Zhou $^{\text {a, b }}$, He Jiang ${ }^{\text {a, } *}$ Zhe Wang ${ }^{\text {a }}$, Xiaoxia Yang ${ }^{\mathrm{c}}$, Erhui Geng ${ }^{\mathrm{a}}$ \\ ${ }^{\text {a }}$ School of Resources and Environment, University of Electric Science and Technology of China (UESTC) \\ ${ }^{\mathrm{b}}$ Institute of Remote Sensing Big Data, Big Data Research Center, UESTC, 2006 Xiyuan Avenue, West Hi-tech Zone, Chengdu, \\ 611731, China \\ ${ }^{c}$ College of Earth Sciences, Chengdu University of Technology, Chengdu, 610059, China \\ *Corresponding author: 1004326962@qq.com
}

\section{Commission VI, WG VI/4}

KEY WORDS : Lands at TM, topographic correction, Cosine correction, C correction, SCS correction, SCS+C correction, snow cover, NDSI

\begin{abstract}
:
The accuracy of snow cover information extraction in remote-sensing images dependent on a variety of factors, especially in mountain area with complex terrain. This paper aims at analyzing the accuracy of snow cover information extraction from remotesensing images, using Landsat5 TM images and DEM data, with the study area of Xinjiang Tianshan, measuring topographic correction effects of Cosine correction, C correction, SCS correction, and SCS + C correction from four aspects: visual comparison, standard deviation, correlation analy sis and histogram, then extract snow cover area for study area. Results showed that $\mathrm{C}$ correction and SCS+C correction performed better among four classic terrain correction models, which indicated changes in snow pixel rat io after correction with variation range of $2 \%$, and correlation coefficient of each band is highest before and after correction.
\end{abstract}

\section{INTRODUCTION}

Snow is solid state of water resources on earth, and plays an important role in human life. In mountainous areas with complex terrain, due to the influence of terrain shield and occlusion, surface features are badly damaged, so it is difficult to extract snow area accurately. Therefore, many researchers have built various topographic correction models to reduce or eliminate the influence of mountainous factors in remote sensing images. These models can be generally classified into three kinds: (1) empirical model, such as band ratio model, Minnaert correction model and empirical statistical correction model. The main advantages of empirical model are simple, less parameters and strong applicability, but its theoretical basis is lack of completeness and enough physical mechanism, with poor representativeness. (2) The physical model, such as Cosine correction, SCS (Sun-Canopy-Sensor) correction model, Proy correction model, Sandmeier correction model. Physical model is established based on the radiative transfer theory, theory basis is perfect, but model is usually nonlinear, complex with many input parameters. (3) The semi-empirical model, such as C correction model, SCS+C correction model. Semi-empirical model combines the advantages of empirical model and physical model, the model parameters usually are empirical parameters with a certain physical meaning.
For different research areas or different experimental data, the test results of the same kind of correction method may be different. This paper, by comparing four typical terrain correction methods of Cosine correction, C correction, SCS correction and $\mathrm{SCS}+\mathrm{C}$ correction, try to find out the suitable methods for Xinjiang Tianshan region, and use it in NDSI snow extraction, in order to study the changes of snow cover area, to improve the accuracy of the snow cover extraction.

\section{FOUR TYPICAL TERRAIN CORRECTION METHODS}

\subsection{Cosine Correction Model}

The Cosine correction model is a simple photometric function that, in the case of illumination not originating from the zenith, normalizes the reflectance of any pixel based on the assumption that the total irradiance received at a pixel is directly proportional to the cosine of the incidence angle $(i)$ as.

$$
\begin{gathered}
L_{H}=L_{T}\left[\frac{\cos \left(\theta_{s}\right)}{\cos (i)}\right] \\
\cos (i)=\cos \left(\theta_{s}\right) \cos S+\sin S \sin \left(\theta_{s}\right) \cos \left(\varphi_{0}-A\right)
\end{gathered}
$$

* Corresponding author: He Jiang, E-mai:1004326962@qq.com 
Where $\theta_{s}$ is the solar zenith angle, $A$ is aspect, $S$ is slope, $\varphi_{0}$ is the solar azimuth angle, $L_{H}$ is the normalized reflectance, $L_{T}$ is the uncorrected reflectance. However, the Cosine model does not take into account diffuse irradiance from atmospheric and terrain sources. As a result, areas that are weakly illuminated by direct irradiance can still receive a considerable proportion of diffuse radiation and are therefore brightened excessively by the Cosine correction. This overcorrection is most pronounced at angles of incidence approaching $90^{\circ}$, where the correction factor becomes very large.

\subsection{Correction Model}

C correction model is more widely used terrain correction model. To correct overcorrection of Cosine correction model, Teilletet al. proposed the addition of a semi-empirical moderator $\mathrm{C}$ to the Cosine correction model, the $\mathrm{C}$ correction model is put forward:

$$
\begin{gathered}
L_{H}=L_{T}\left[\frac{\cos \left(\theta_{s}\right)+C}{\cos (i)+C}\right] \\
\cos (i)=\cos \left(\theta_{s}\right) \cos S+\sin S \sin \left(\theta_{s}\right) \cos \left(\varphi_{0}-A\right)
\end{gathered}
$$

Based on an examination of image data, a linear relationship exists between $L$ and $\cos (i)$ in the form:

$$
L=a^{*} \cos (i)+b
$$

The parameter $\mathrm{C}$ is a function of the regression slope (b) and intercept (a) $\mathrm{C}=\mathrm{b} / \mathrm{a}$.

C correction model avoid the overcorrection problems of Cosine correction model in shadow areas to some extent. C coefficient reduces the overcorrection phenomenon in shadow area by increasing the value of the denominator in the model to restrain topographic influence, but the coefficient is lack of clear physical interpretation. In fact, the above linear relationship is only suitable for special surface, for mountains, this linear relationship is inadequate. From this point, $\mathrm{C}$ correction model also exist obvious limitation.

\subsection{SCS Correction Model}

For mountains with vegetation, Gu proposed SCS (SunCanopy-Sensor) correction model. The SCS correction is equivalent to projecting the sunlit canopy from the sloped surface to the horizontal, in the direction of illumination. Assuming that the reflected radiation from the sunlit canopy is largely independent of topography due to the geotropic (vertical) nature of tree growth, the integrated reflectance from the sunlit canopy is proportional to its area.

$$
\begin{gathered}
L_{H}=L_{T} \frac{\cos S \cos \theta_{S}}{\cos i} \\
\cos i=\cos \left(\theta_{s}\right) \cos S+\sin S \sin \left(\theta_{s}\right) \cos \left(\varphi_{0}-A\right)
\end{gathered}
$$

The geometric relationship between the sun and the tree canopy keeps same before and after correction in SCS Correction Model, so it is more in line with the actual situation of trees geotropism growth, suitable for the terrain correction of high vegetation coverage areas. Due to SCS Correction Model also ignores the influence of the scattered radiation from the sky and the surrounding terrain, the shadow areas in the image exist overcorrection.

\subsection{SCS +C Correction Model}

The cause of the overcorrection in the SCS model is similar to that with the Cosine correction. Scott introduced $\mathrm{C}$ coefficient on the basis of SCS correction model, proposed SCS+C correction model:

$$
L_{H}=L_{T} \frac{\cos S \cos \theta_{S}+C}{\cos i+C}
$$

Parameter $\mathrm{C}$ is intended to be an improvement to the SCS correction in a similar way as the $\mathrm{C}$-correction improves on the Cosine correction. The parameter $\mathrm{C}$ was chosen due to its past success in moderating the Cosine correction. While other parameters that account for diffuse atmospheric and terrain radiance may provide additional refinements, the computational costs and input parameter requirements may be impractical and not consistent with the fundamental philosophy of general applicability of the SCS model as proposed by Gu and Gillespie.

\section{EXPERIMENT AND ANALYS IS}

\subsection{Research Region Introduction}

The snow water resources of Xinjiang accounts for one third of the total amount of snow water in China, Tianshan snowfall is abundant in winter, which is the birthplace of many rivers in Xinjiang. The climate of the complex terrain of this region is cold and wet, which has the high altitudes snow space-time distribution ,a large number of snowmelt-runoff also often lead to flooding, the influence of regional climate, natural environment and human activity impact cannot be ignored, so monitoring and assessment of snow and analyzing its influence is very necessary.

\subsection{Topographic Correction}

Experimental data mainly includes Landsat5 TM images, shown in Figure I (a) (5, 4, 2 band synthetic a false-color image, LT514403020111114KHC00, track number 144/30, solar azimuth $142.3931^{\circ}$, solar altitude $54.4263^{\circ}$, pixel size $30 \mathrm{~m} * 30 \mathrm{~m})$, and DEM, shown in Figure I (b) (ASTGTM2_N43E085, from "International Scientific \& Technical Data Mirror Site, Computer Network Information Center, Chinese Academy of Sciences", resolution 30m) in Tianshan Mountains.

The data procedure is shown in Figure 2, which includes TM data processing and DEM data processing. TM data processing includes five steps: radiation calibration, converting to data BIL, 
FLAASH atmospheric correction, resizing the same region; DEM data processing includes resizing the same region and extracting the slope and aspect. The slope is shown in Figure 3(a) and the aspect is shown in Figure 3(b). Because snow cover extracting just needs $\mathrm{TM}$ band 2, 4, 5, so just do terrain correction for the three bands. According to the principle described above, the topographic corrections are realized by MATLAB programming, the results of Cosine correction (Figure 4(a)), C correction (Figure 4(b)), SCS correction (Figure 4(c)), and SCS+C correction (Figure 4(d)), are shown in Figure 4.

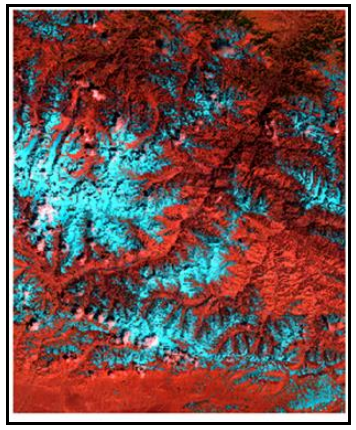

a. TM image (band 5, 4, 2)

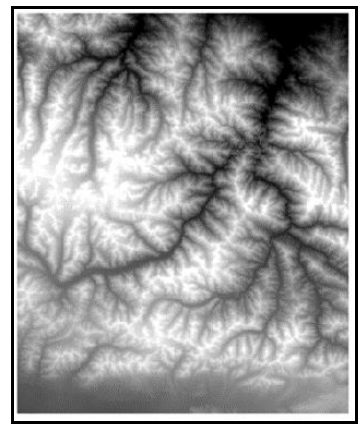

b. DEM
Figure 1. TM and DEM data

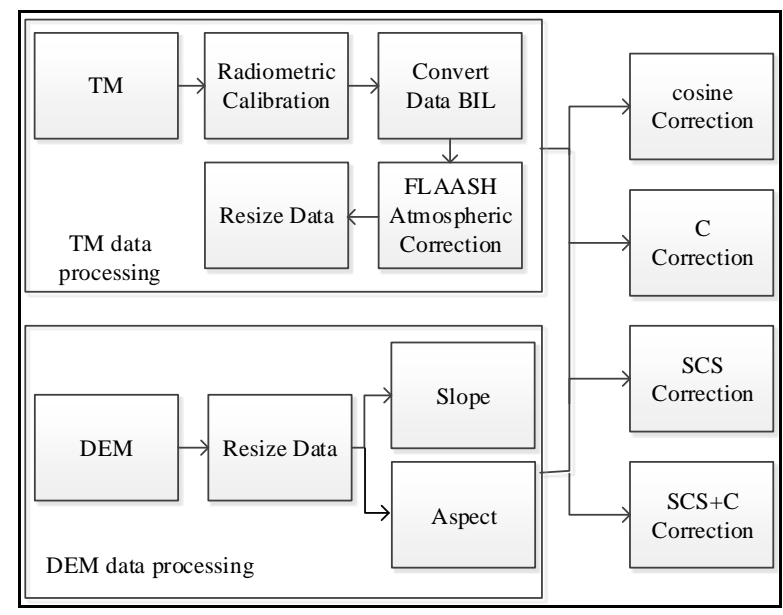

Figure 2. Experiment data procedure flow diagram

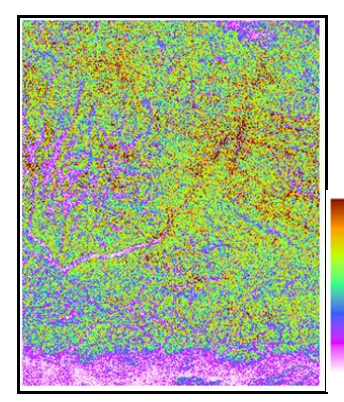

a. Slope

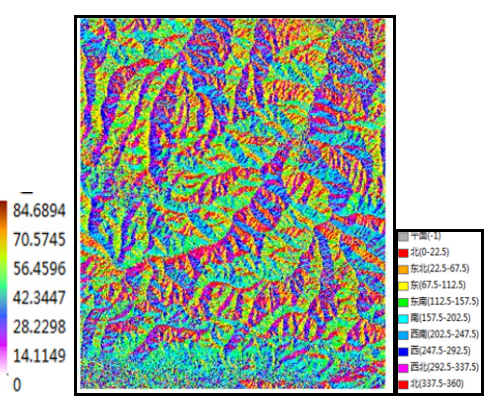

b. Aspect
Figure 3. Slope and aspect

\subsection{Analysis of Terrain Correction Results}

Evaluating topographic correction always takes three aspects into consideration as follow: (1) Visual comparison, changes of stereoscopy can be viewed via false color and produced intuitive correction effect; (2) Statistical characteristics analysis, on the basis of research by Justice, Richter, and Shepherd, changes on standard deviation after correction can measure correction effects quantificational, but is not able to reflect effects of terrain correction most intuitionally; (3)Analy sis of illumination coefficient and correlation of pixel's value, in general, the brightness value of original images in mountain area is correlated with illumination coefficient while terrain correction aims to reduce that correlation in order to reduce influences of terrain, and also correlation coefficient between images brightness value and illumination coefficient reflects terrain factors effects on radiation value. So this standard can measure correction effects better and universal. This paper did the analysis from visual comparison, regression analy sis, histogram and changes of snow cover images before and after correction.

\subsubsection{Visual Comparison}

Comparing original images composited by false color to those corrected (Figure 4), images were more flat, less stereoscope, showing more details involved in shadow area, eliminating terrain change information. Comparing reflectance of ground objects on shady slope to those on sunny slope, the former gets higher and the latter gets lower. And yet because of the low spatial resolution of DEM, slope and aspect figured out have error, so there are overcorrection in mountain ridge and valley. In addition, registration error between DEM and original images and DEM internal error have influences on correction effect.

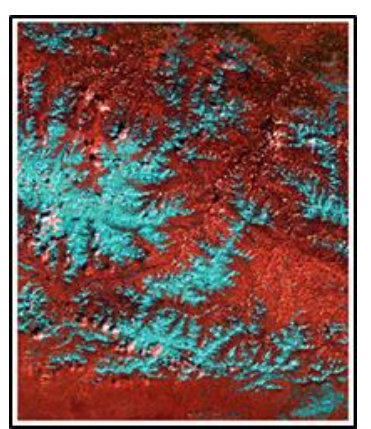

a. Cosine correction

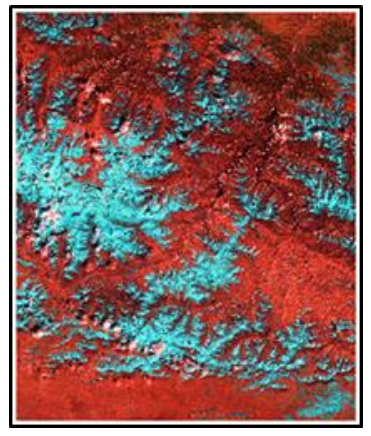

c. SCS correction

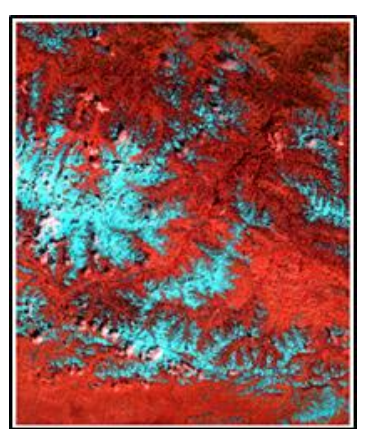

b. C correction

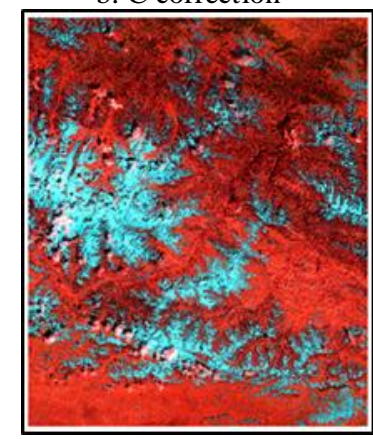

d. $\mathrm{SCS}+\mathrm{C}$ correction
Figure 4. Comparison after correction (band 5, 4, 2) 


\subsubsection{Regression Analysis}

Figure 5 shows that standard deviation of each band changed in varying degrees: values increasing most obviously after Cosine correction, so does SCS correction, and there are less obvious descents in $\mathrm{C}$ and $\mathrm{SCS}+\mathrm{C}$ correction results but not so obviously, which indicated that brightness change is weakened, brightness difference was reduced, and terrain influences is eliminated partly. In Cosine correction model, images brightness varies sharply, which is overcorrection. Therefore, $\mathrm{C}$ correction and SCS+C correction work better comparatively.

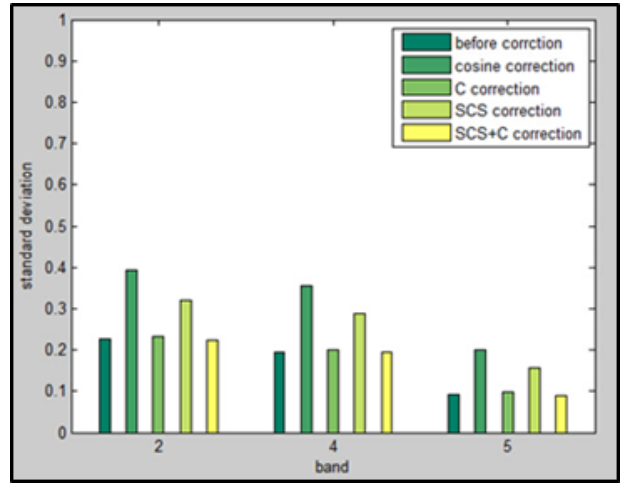

Figure 5. Standard deviation

\begin{tabular}{|c|c|c|c|}
\hline band & $\mathbf{2}$ & $\mathbf{4}$ & $\mathbf{5}$ \\
\hline Cosine correction & 0.6547 & 0.5792 & 0.3820 \\
\hline C correction & 0.9943 & 0.9847 & 0.8547 \\
\hline SCS correction & 0.7195 & 0.6551 & 0.4541 \\
\hline SCS+C correction & 0.9954 & 0.9877 & 0.8940 \\
\hline
\end{tabular}

Table 1. The band pertinence and after correction

Table 1 shows that there are high correlation between original images and those corrected in the ways of $\mathrm{C}$ and $\mathrm{SCS}+\mathrm{C}$ in all bands, in contrast, those in Cosine correction and CSC correction are lower with different correlation coefficients. In test of three bands, the correlation coefficient of band 2 corrected by $\mathrm{SCS}+\mathrm{C}$ and corresponding original images was the highest, reaching 0.9954 , closing to 1 . By contrast, correlation of $\mathrm{SCS}+\mathrm{C}$ correction performed best.

\subsubsection{Histogram Comparison}

Using histogram can parse out the regularity of data, more intuitive to see the quality of the product characteristics of distribution. The intensity of the image histogram describes the image of each value (Digital Number, DN) the number of pixels and statistical distribution, is an important index to evaluate the quality of images. By observing the crests and contrast, can just the quality of images. It shows there exits some extent of stretching after the Cosine correction, C correction, SCS correction, SCS + C correction, the crest is scattered of Cosine correction and SCS correction, but the crest of $\mathrm{C}$ correction and $\mathrm{SCS}+\mathrm{C}$ correction is dense, but for band 5 appears double crests phenomenon. By contrast, $\mathrm{C}$ correction and $\mathrm{SCS}+\mathrm{C}$ correction are better, but is not normal type, that is, is not stable, do not conform to the low, middle and high on both sides, around the approximate symmetrical features.

\subsection{Snow Cover Extraction}

Different material and the same material under different conditions, the reflection and radiation characteristics are different, which is the basic principle of remote sensing. Snow monitoring is based on different reflectance among snow, bare land, cloud, different depth of snow. As shown in Figure VI, both snow and cloud have their own unique spectrum reflection and radiation characteristic. The method of Normalized Difference Snow Index (NDSI) is applied broadly, named SNOMAP, the integral part is NDSI. For Landsat TM data:

$$
N D S I=\left(b_{2}-b_{5}\right) /\left(b_{2}+b_{5}\right)
$$

Where $b_{2}$ and $b_{5}$ respectively prefer to the reflectivity of the band $2(0.52 \sim 0.60 \mu \mathrm{m})$ and band $5(1.55 \sim 1.75 \mu \mathrm{m})$ of Landsat TM images, with the spatial resolution of $30 \mathrm{~m}$. NDSI values greater than or equal to approximately 0.4 were found to represent snow cover. Water bodies may have NDSI values in the range of those for snow; however, water has a lower nearinfrared reflectance and thus can be distinguished from snow.

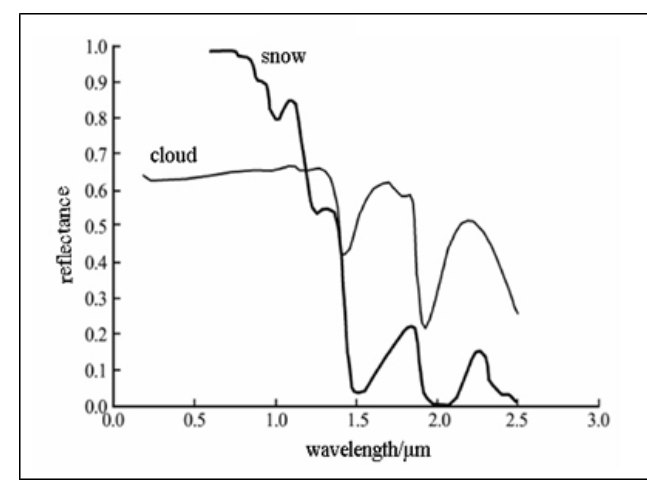

Figure 6. Cloud and snow reflection characteristics
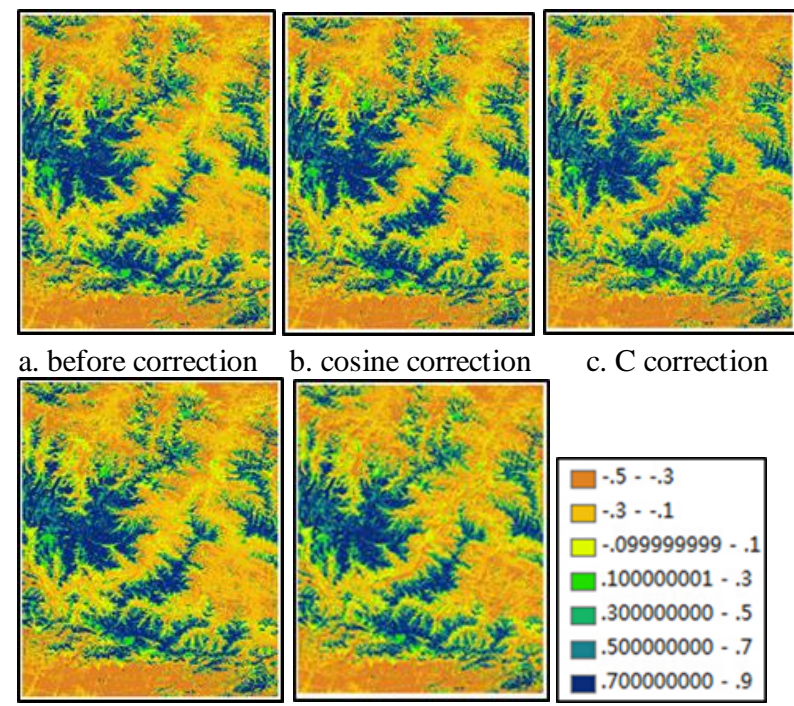

c. C correction

d. SCS correction e. SCS+C correction

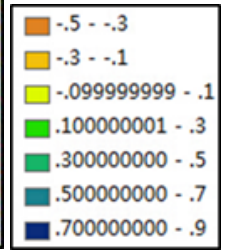

Figure 7. NDSI comparison and after topographic correction 
In SNOMAP, an additional test for snow must be conducted after the NDSI is calculated. In order to be mapped as snow in SNOMAP, the TM band 4 reflectance must be $>11 \%$. A pixel is classified as snow covered if results of both the NDSI and reflectance tests lie within the intersection. Hall pointed out that if the MODIS band 4 reflectance is $<10 \%$, then the pixel will not be mapped as snow even if the other criteria are met. A pixel is mapped as snow when meet the three conditions.

Accurate detection of snow cover change is very important, and the terrain is one of the important factors affecting the accuracy. In order to further evaluate the effect of the topographic correction, extract snow cover to the images before and after terrain correction through calculating NDSI, and NDSI images and snow cover images are shown in Figure VII and Figure VIII respectively: white represents snow pixels, black represents areas without snow. Table II gives the number and proportion of snow pixels before and after correction, we can see that snow proportion become big after Cosine and SCS correction, but range is not big, within $2 \%$; snow proportion become small after $\mathrm{C}$ and $\mathrm{SCS}+\mathrm{C}$ correction within $1.5 \%$.

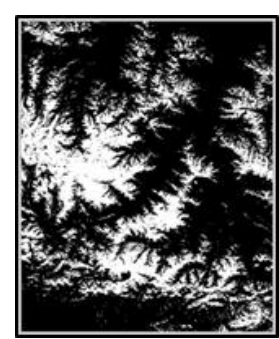

a. snow cover

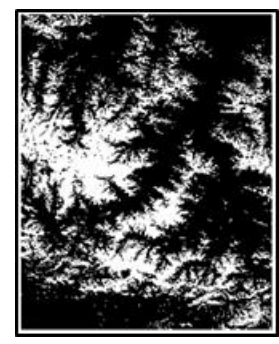

d. SCS correction

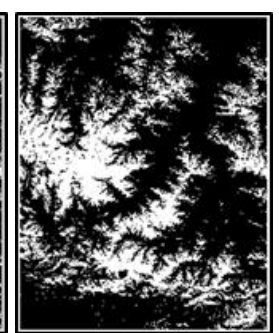

b. Cosine correction

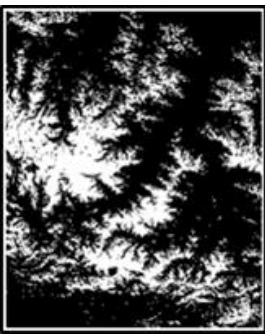

e. $\mathrm{SCS}+\mathrm{C}$ correction

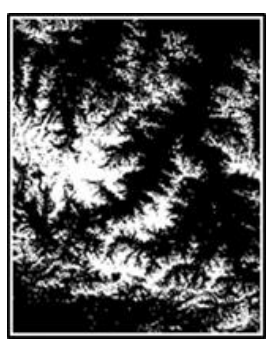

c. C correction
Figure 8. Snow cover comparison before and after topographic correction

\begin{tabular}{|l|l|l|l|l|l|}
\hline & $\begin{array}{l}\text { Before } \\
\text { correction }\end{array}$ & $\begin{array}{l}\text { Cosine } \\
\text { correction }\end{array}$ & $\begin{array}{l}\text { C } \\
\text { correction }\end{array}$ & $\begin{array}{l}\text { SCS } \\
\text { correction }\end{array}$ & $\begin{array}{l}\text { SCS+C } \\
\text { correction }\end{array}$ \\
\hline $\begin{array}{l}\text { Snow } \\
\text { pixels }\end{array}$ & 1987452 & 1987452 & 1871889 & 1985265 & 1937536 \\
\hline $\begin{array}{l}\text { Snow } \\
\text { ration }\end{array}$ & $28.394 \%$ & $28.555 \%$ & $26.895 \%$ & $28.524 \%$ & $27.838 \%$ \\
\hline
\end{tabular}

Table 2. Snow cover ration before and after topographic correction

\section{CONCLUSION}

As the influence of topography, topographic effects restrict the accuracy of snow cover information extraction of remote-sensing images, especially in mountain area with complex terrain. The terrain correction can solve this problem well and make remote sensing data presents information more accurately. This paper used Cosine correction, C correction, SCS correction and SCS + $\mathrm{C}$ correction to correct TM images of Tianshan, analyzing the difference between before and after the correction through visual comparison, standard deviation, correlation analysis and histogram, and applying this to SNOMAP snow cover extraction. Four typical topographic correction methods all made senses on topographic effects, but $\mathrm{SCS}+\mathrm{C}$ correction worked better compared to other three ways, but there were difference to real ground surface reflectance. Therefore, studying more efficient models for terrain correction will be carried on in the future.

\section{ACKNOWLEDGEMENTS}

This research supported by National Natural Science Foundation of China (No. 41471332, 41101354 and 41201440), and the National High Technology Research and Development Program of China (No. 2013AA12A302). The Fundamental Research Funds for the Central Universities (No.ZYGX2011J077), and Fund of China Scholarship Council.

\section{REFERENCES}

Colby J D, Keating P L, J., 1998. Land cover classification using Landsat TM imagery in the tropical high land: the influence of anisotropic reflectance. International Journal of Remote Sensing, 19 (8): 1479.

Cavayas F, J., 1987. Modeling and correction of topographic effect using multi-temporal satellite images. Canada Journal of Remote Sense, 13 (2): 49.

Duguay C R , Le Drew E F, J., 1992. Estimating surface reflectance and albedo from Landsat TM over rugged terrain. Photogrammetric Engineering and Remote Sensing, 58: 551.

Gu D, Gillespie A, J., 1998. Topographic normalization of Landsat TM images of forest based on sub-pixel sun canopy sensor geometry. Remote Sense of Environment, 64: 166.

Hao Pei, Jingwei Han, J., 1994. Research of using meteorological satellite to monitor snow. Resources and Environment in arid areas, 8(26): 446-452.

Itten K I, Meyer P, J., 1992. Geometric and radiometric correction of TM data of mountainous forested areas. IEEE Transaction on Geoscience and Remote Sensing, 31 (4):764.

Liming Wang, Qingiu Tian, Wei Cheng, C., 2007. The terrain correction method contrast research //Academic exchange on the 16th national remote sensing technology, 2007: 80-82.

Meyer P, It ten K I, Kellenberger T, et al, J., 1993. Radiometric corrections of topographically induced effects on Landsat TM data in an alpine environment. ISPRS Journal of Photogrammetry and Remote Sensing, 48: 17. 
Riano D, Chuvieco E, Sales J, Aguado I, J., 2003. Assessment of different topographic corrections in Landsat TM data for mapping vegetation types. IEEE Transactions on Geoscience and Remote Sensing, 41:1056-1061.

Scott A, Soenen, Derek R, J., 2005. SCS + C: a modified suncanopy-sensor topographic correction in forested terrain $[\mathrm{J}]$. IEEE Transaction on Geoscience and Remote Sensing, 43(9): 2148.

Shepherd J D, Dymond J R, J., 2003. Correcting satellite imagery for the variance of reflectance and illumination with topography. International Journal of Remote Sensing, 24: 35033514.

Teillet P M, Guindon B, Goodeonugh D G, J., 1982. On the slope-aspect correction of multi-spectral scanner data. Canada Journal of Remote Sense, 1982 (8): 84.

Wei Huang, Liangpei Zhang, Pingxiang Li, J., 2005. An improved satellite images of terrain correction algorithm. Journal of Image and Graphics, 10(9): 1124.

Yaowu Zhong, D., 2006. Beijing mountainous terrain image radiation correction methods research. Beijing: School of geography and remote sensing science, Beijing normal university. 